\title{
The genius of Roger Stanier
}

\author{
Morris Goldner PhD
}

$\mathrm{T}$ he esteem for the scientific activity of Roger Stanier has been widespread. Apart from the oral and written history, an exploration of the archives of the Pasteur Institute in Paris and the personal Stanier papers in the National Archives of Canada in Ottawa fulfills this admiration. More recently, an institute has been formed in Canada that honours the name and memory of Roger Stanier with the consent of the Stanier family. English and French were the languages used by Dr Stanier in his work during his lifetime. The present commentary brings to the foreground the essence of his thinking, which is as relevant today as it was in his time. The condensed description serves as a commemoration so that it will not be forgotten that Roger Stanier was a Canadian as well as a world-renowned scientist.

Roger Stanier (1916-1982) was a profound scholar whose studies spanned many years and concerned the basic tenets of evolution and adaptation. This distinguished Canadian-born (Victoria, British Columbia) scientist first embarked on the study of microorganisms many decades ago and spent his working life as a microbiologist in the United States and later in France. Early on, he spent a period of research on bacterial metabolism and physiology with modern pioneer microbiologist Marjorie Stephenson through a Guggenheim fellowship at Cambridge University (United Kingdom). Beginning in 1947, he devoted 24 years to the University of California at Berkeley (USA) where he attained the position of professor and chairman. During his years at Berkeley, Stanier made many fundamental contributions to our understanding of the microbial world. In 1957, with outstanding collaborators, he produced what was to become the leading textbook on microbiology during his time, entitled The Microbial World (traduit en francais et édité par Masson en 1966). This text was subsequently published in five editions over 30 years. In 1971, the Stanier family moved to France where Dr Stanier and his wife, Germaine, a French microbiologist and a distinguished scientist in her own right, together joined the Pasteur Institute. Roger Stanier assumed the position of 'chef de service' of the microbial physiology unit. Throughout his life, he maintained his Canadian citizenship.

From his early days, taxonomy was a constant challenge to Stanier and his interest in the subject drew him into the Bergey's Manual Trust (USA) during the years of its gestation. The Bergey's Manual Trust developed into the scholarly authority for species recognition. Roger Stanier did much for its character and content, which gave him a wide view of microbiology and his interest evolved on a broad scale. His brilliant effort to bridge the gaps in our knowledge of complex evolutionary development helped characterize the microbial world.
By depicting the course of evolution in terms of efficient endocytosis, Stanier directed attention to organellar structures and their eventual symbiotic relationships. He explained that the variety of cytoplasmic structures bearing colour pigments actually reflected ancient evolutionary diversity. Photopigment synthesis of free-living forms would have been preserved in the photosynthetic organelles. In time, nuclear organization prevented the evolutionary paths of cosymbionts from freely going their own way. His exceptional insight led him to appreciate the evolutionary significance of bacterial photosynthesis; it was the basis by which he traced the adaptation of organisms from anoxygenic (anaerobic) to oxygenic (aerobic) lifeforms. This produced a turning point in evolution when he realized that pigments in microorganisms played the important role of trapping energy from light. Selection in the emerging eukaryotic cell would have centred on improved efficacy of predation. He then fathomed that special relationships of microbial groups with unique physiological properties tend to occur to establish a shared opportunity for their survival.

When Stanier turned his attention to the habitat of microorganisms to explain the selective pressures in a natural environment, he was able to foresee the connection between symbiotic relationships and microbial ecology. He was concerned that real changes would not be recognizable except under authentic environmental conditions that ensured the significant connections in the microenvironment. Stanier understood that the key to change was the availability of energy and diversity of mechanisms for its generation.

Roger Stanier's intense interest in adaptation concerned multiple simultaneous adaptation in enzyme formation conceived as a systematic format at a basic biochemical level. The presence of a control mechanism was considered a stable character, which eventually showed that pathways could be regulated and that the control would in turn be conserved for this purpose in a particular biological group. He not only systematically clarified pathway metabolism, he also provided the basis on which the linkage to other biochemical pathways could be envisioned. It illustrated alternative pathways and detailed proof of microbial behaviour in a particular biochemical niche.

In emphasizing evolution and adaptation, Stanier described the organic environment as consisting of both systematic and fluctuating occurrences, thus enabling organisms to move toward successive adaptation. He defined systematic variations as those that involved gradual unidirectional and fluctuating variations as short-term changes. Systematic events dealt with molding of the genotype, allowing for an organism to be selected to fit conditions of its environment in a geological time slot.

Brock University, St Catharines, Ontario; Universite Laval, Quebec City, Quebec

Correspondence and reprints: Dr Alain Dublanchet, Centre Hospitalier Intercommunal de Villeneuve-Saint-Georges, Lab de Microbiologie,

40 Allée de la Source, Villeneuve-Saint-Georges, Cedex 94195, France. Telephone 01-43-86-22-14, e-mail adublanchet@noos.fr

Received for publication November 14, 2006. Accepted January 8, 2007 
Fluctuating events dealt with the change during a period of observation that was just sufficient to assess and pinpoint any pattern in those events that permitted possible selection of different genotypes.

However, even with successive adaptation in nature, he recognized that any mutation could result in a disaster if it interfered with the competitive edge of an organism. Hence, an organism must retain that certain array of genes that gives it maximum fitness to ensure its environmental survival. Photopigments are of great biological significance, their importance being to permit the coexistence of different kinds of photosynthetic organisms in one habitat. Their ability to absorb light from certain regions of the solar spectrum benefited the coexisting symbionts. Thus, Stanier formulated the ingredients for analyzing symbiosis according to the degree of intimacy, the balance of advantage and the extent of dependence of each symbiont. The concurrent development in genetic structure led to a more consistent gene makeup.

Roger Stanier's approach was highly influential because he thought that the difficult questions facing biology should also include microorganisms. By and large, Stanier brought microbiology into the mainstream of the biological sciences. He became renowned for his enormous studies on the fundamentals of microbiology and related biochemistry. His pioneering research concerned enzyme adaptation, the role of pigments, the assimilation of components with regard to chlorophyll, the oxidative processes of aromatic compounds, the comparative biochemistry and evolution of microorganisms and the establishment of blue-green algae as cyanobacteria. This interest in blue-green algae stemmed from his earlier passion for taxonomy and photosynthesis, and the need to determine the distinction between prokaryotes and higher microbial eukaryotes. In the novel investigation of the blue-green algae, Stanier and his wife, Germaine, stressed structure and function relationships, receiving overwhelming substantiation from increasing microscopy studies.

Over his lifetime, Stanier received many awards and much international recognition including the Chevalier de la Légion
d'Honneur (1977), Fellow of the Royal Society-London (1978) and also Foreign Associate of the National Academy of Sciences (1979).

The year 2006 marks 90 years since Roger Stanier's birth and 2007 notes 25 years since his passing. The year 2007 also marks 50 years since the appearance of his influential text, The Microbial World, published in 1957, which resulted in five editions. What a compelling constellation of events in the life and work of Roger Stanier!

ACKNOWLEDGEMENTS: The authors appreciate the studies of Josephine Accaputo-Gendron and Morris Goldner, 'Roger Stanier: Diversity as the key to a new era for biology. Perspect Biol Med 1993;37:48-54', that facilitated this condensed treatise on the substance of Stanier's life and work, and that resulted in the extract reprinted with permission of the Johns Hopkins University Press.

\section{RECOMMENDED READINGS}

1. Stanier RY. Adaptation, evolutionary and physiological: Or

Darwinism among the microorganisms. In: R Davies, EF Gale, eds. Adaptation in Microorganisms, Third Symposium of the Society for General Microbiology. Cambridge: Cambridge University Press, 1953.

2. Stanier RY, Cohen-Bazire G. The role of light in the microbial world; some facts and speculations. In: Williams REO, Spicer CC, eds. Microbial Ecology, Seventh Symposium of the Society for General Microbiology. Cambridge: Cambridge University Press, 1957.

3. Stanier RY, Doudoroff M, Adelberg EA. The Microbial World, 1st edn. New Jersey: Prentice-Hall, 1957.

4. Canovas JL, Ornston LN, Stanier RY. Evolutionary significance of metabolic control systems. The beta-ketoadipate pathway provides a case history in bacteria. Science 1967;156:1695-9.

5. Stanier RY. Some aspects of the biology of cells and their possible evolutionary significance. In: Charles HP, Knight BCJG, eds. Organization and Control in Prokaryotic and Eukaryotic Cells, Twentieth Symposium of the Society for General Microbiology. Cambridge: Cambridge University Press, 1970.

6. Stanier RY. The journey, not the arrival matters. Ann Rev Microbiol 1980;34:1-48.

7. Stanier RY, Ingraham JL, Wheelis ML, Painter PR. The Microbial World, 5th edn. New Jersey: Prentice-Hall, 1986. 


\title{
Le génie de Roger Stanier
}

\author{
Alain Dublanchet MD
}

L 'admiration portée à Roger Stanier pour son activité scientifique a été largement partagée de son vivant. Autant par l'histoire orale et écrite que par l'étude des documents conservés à l'Institut Pasteur de Paris ou par la lecture des papiers personnels de Stanier aux Archives nationales du Canada à Ottawa, cette estime ne peut qu'être confirmée aujourd'hui. Récemment, un institut canadien a honoré, avec le consentement de la famille, le nom et la mémoire de Roger Stanier. L'anglais et le français étaient les langues pratiquées par Roger Stanier dans l'exercice de son métier. La profondeur de ses pensées est aussi pertinente aujourd'hui que de son vivant. Ce bref rappel se propose de commémorer Roger Stanier, de sorte qu'on n'oublie pas qu'il était tout autant un Canadien qu'un scientifique mondialement renommé.

Roger Stanier (1916-1982) était un savant accompli dont les études se sont étalées de nombreuses années et ont concerné les principes de base de l'évolution et de l'adaptation. Ce remarquable scientifique d'origine canadienne (Victoria, Colombie-Britannique) s'est engagé dans l'étude des microorganismes et a passé sa vie professionnelle comme microbiologiste, d'abord aux États-Unis, puis en France. Il a commencé par une période de recherche sur la physiologie et le métabolisme bactériens avec l'initiatrice de la microbiologie moderne, Marjorie Stephenson, avec le soutien d'une bourse universitaire Guggenheim de l'université de Cambridge (Royaume-Uni). A partir de 1947, il a consacra 24 ans à l'université de Berkeley en Californie où il a occupa les postes de professeur puis de directeur de département. Durant ses années à Berkeley, Stanier a apporta de nombreuses contributions fondamentales à notre compréhension du monde microbien. En 1957, avec d'éminents collaborateurs, il a produit ce qui allait devenir le principal manuel de microbiologie de son époque, The Microbial World. Ce livre a été ultérieurement réédité cinq fois en 30 ans, traduit et édité en français par Masson en 1966. En 1971, Stanier et sa famille sont allés en France où, lui et son épouse, Germaine, microbiologiste française et éminente scientifique dans sa propre spécialité, ont rejoint ensemble l'Institut Pasteur. Roger Stanier occupa le poste de chef de service de l'unité microbienne de physiologie. Durant toute sa vie, il a conservé la citoyenneté canadienne.

Pour Stanier, dès le début, la taxonomie a été un défi constant, et son intérêt pour le sujet l'a conduit vers l'équipe du Bergey's Manual pendant les années de son élaboration. Le
Bergey's Manual s'est imposé comme la référence pour l'identification des espèces. Roger Stanier a fait beaucoup pour le caractère et le contenu de l'ouvrage, lui assurant une vaste vue sur la microbiologie et élargissant ses intérêts. L'effort soutenu dans son travail pour porter à notre connaissance les mécanismes complexes du développement de l'évolution a aidé à caractériser le monde microbien.

En décrivant le cours de l'évolution en termes d'endocytose efficace, Stanier a attiré l'attention sur la structure des organelles et leurs éventuelles relations symbiotiques. Il a montré que la diversité des structures cytoplasmiques à l'origine de la couleur des pigments, reflétait avec précision la diversité passée de l'évolution. La synthèse photopigmentaire des formes libres a été préservée dans les organelles photosynthétiques. À l'origine, l'organisation du noyau a contrarié la liberté d'évolution des symbiotes. La perspicacité exceptionnelle de Stanier l'a mené à apprécier l'importance de la photosynthèse bactérienne dans l'évolution. C'est la base par laquelle il a suivi l'adaptation des organismes anoxygéniques (anaérobies) vers les formes de vie oxygéniques (aérobies). Il s'est rendu compte que les pigments des microorganismes ont joué un rôle important dans la capture de l'énergie lumineuse, ce qui a constitué un tournant décisif de l'évolution. Le choix par la cellule eucaryote naissante se serait porté sur la meilleure efficacité de la fixation. Il a alors envisagé que les rapports spécifiques des groupes microbiens ont tendance à se produire avec des propriétés physiologiques uniques, afin d'assurer des possibilités commune de survie.

Quand Stanier a porté son attention sur l'habitat des microorganismes pour expliquer les pressions sélectives dans un environnement normal, il a pu anticiper le lien entre les rapports symbiotiques et l'écologie microbienne. Il craignait qu'on ne puisse reconnaître de vrais changements sauf dans des conditions environnementales réelles comportant les rapprochements significatifs de l'environnement. Stanier avait compris que la clef du changement était la disponibilité de l'énergie et la diversité des mécanismes pour sa formation.

Le grand intérêt de Roger Stanier pour l'adaptation portait sur l'adaptation multiple et simultanée de la formation d'enzymes conçue comme un processus systématique biochimique de base. La présence d'un mécanisme de régulation était considérée comme un caractère stable, qui par la suite a montré que les voies pouvaient être régulées et que le 
mécanisme tendait à son tour à être conservé dans ce but au sein d'un groupe biologique particulier. Il a non seulement clarifié systématiquement la voie métabolique, mais a également fourni la base sur laquelle le lien avec d'autres voies biochimiques pouvait être envisagé. Il a décrit des voies alternatives et le comportement microbien dans une situation biochimique particulière.

En soulignant l'évolution et l'adaptation, Stanier a décrit l'environnement organique comme la résultante d'événements systématiques et variables, permettant de ce fait à des organismes d'évoluer vers une adaptation progressive. Il a défini les variations systématiques comme celles qui mettaient en jeu des variations unidirectionnelles et fluctuantes graduelles sous forme de changements à court terme. Les événements systématiques ont assuré l'organisation génotypique en tenant compte pour qu'une organisation soit choisie de l'état de l'environnement dans une fenêtre de temps géologique. Les occurrences variables ont permis le changement pendant une période d'observation suffisante pour évaluer et décrire avec exactitude n'importe quel modèle des événements qui permettent le choix possible de différents génotypes.

Cependant, même avec l'adaptation successive dans la nature, il a établi que n'importe quelle mutation pouvait être désastreuse si elle s'opposait à l'avantage concurrentiel d'un organisme. Par conséquent, un organisme doit maintenir l'ordre des gènes lui donnant la forme physique optimum pour lui assurer sa survie dans l'environnement. Les photopigments ont une grande portée biologique, leur importance étant de permettre la coexistence de différentes sortes d'organismes photosynthétiques dans un habitat. Leur capacité d'absorber la lumière de certaines régions du spectre solaire a favorisé la coexistence des symbiotes. C'est ainsi que Stanier a formulé les éléments pour analyser la symbiose en fonction du degré d'intimité, de l'équilibre favorable, et du degré de dépendance de chacun des symbiotes. Le développement simultané de la structure génétique a donné une composition plus cohérente du gène.

La contribution de Roger Stanier a été fondamentale parce qu'il a estimé que les questions complexes en biologie concernaient également les microorganismes. Plus généralement, Stanier a introduit la microbiologie dans le courant dominant des sciences biologiques. Sa renommée provient de l'ampleur de ses vastes études sur les principes fondamentaux de la microbiologie et de la biochimie relative. Ses recherches novatrices portaient sur l'adaptation enzymatique, le rôle des pigments, l'assimilation des éléments en rapport avec la chlorophylle, les processus oxydants des composés aromatiques, la biochimie et l'évolution comparatives des microorganismes ainsi que les fondements des algues bleues comme cyanobactéries. Cette passion pour les algues bleues est née de sa première passion pour la taxonomie et la photosynthèse, et la nécessité de distinguer les procaryotes des eucaryotes microbiens supérieurs. Dans la nouvelle recherche sur les algues bleues, Stanier et son épouse, Germaine, ont souligné les rapports entre la structure et la fonction, en trouvant irrésistiblement la confirmation dans les études microscopiques.

Durant son existence, Stanier reçut de nombreuses récompenses et reconnaissances internationales dont le titre de Chevalier de la Légion d'Honneur (1977), le titre de membre de la Royal Society de Londres (1978) et celui d'associé étranger de la National Academy of Sciences (1979).

L'année 2006 est l'année du 90ème anniversaire de la naissance de Roger Stanier et en 2007 il y aura 25 ans qu'il aura disparu. L'année 2007 marquera également les 50 ans de l'apparition de son ouvrage majeur, The Microbial World, qui fut édité pour la première fois en 1957, réédité cinq fois, traduit et publié en français par Masson. Quel ensemble d'admirables événements que la vie et le travail de Roger Stanier !

REMERCIEMENTS: Les auteurs remercient Josephine Accaputo-Gendron et Morris Goldner, pour leur travail intitulé "Roger Stanier: Diversity as the Key to a New Era for Biology, Perspec Biol Med 1993;37:48-54 » qui leur a facilité cette brève présentation de la vie et du travail de Roger Stanier ainsi que l'éditeur, Johns Hopkins University Press, pour l'autorisation d'utiliser des d'extraits de cette publication.

\section{LECTURES RECOMMANDÉES}

1. Stanier R.Y. La place des bactéries dans le monde vivant. Ann Inst Pasteur (Paris) 1961;101:297-312.

2. Stanier R.Y, Doudoroff M, Adelberg E.A. Microbiologie Générale, Collection Précis de Sciences Biologiques sous la direction de P. P. Grassé, Masson et Cie éditeurs, Paris, 1966:638.

3. Stanier R.Y, Lwoff A. Le concept de microbe de Pasteur a nos jours. Nouv Presse Med 1973;2:1191-8.

4. Stanier R.Y. Réflexions sur la taxonomie de Pseudomonas. Bull Inst Pasteur 1976;74:255-70. 


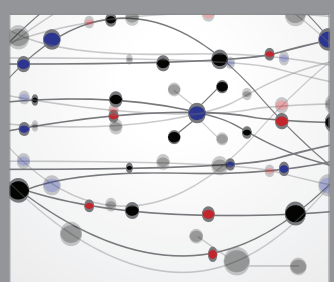

The Scientific World Journal
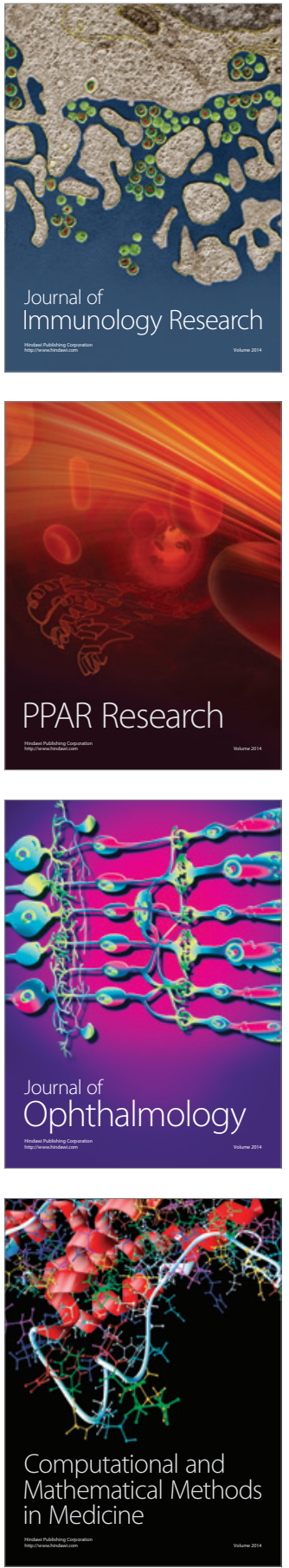

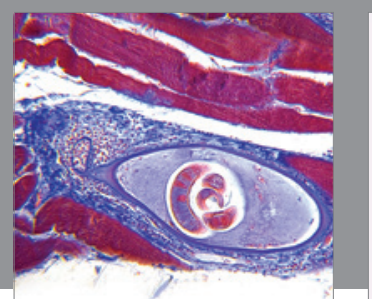

Gastroenterology Research and Practice

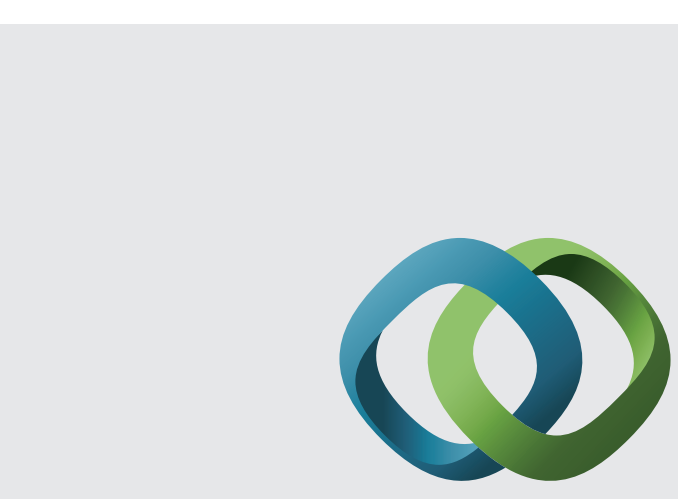

\section{Hindawi}

Submit your manuscripts at

http://www.hindawi.com
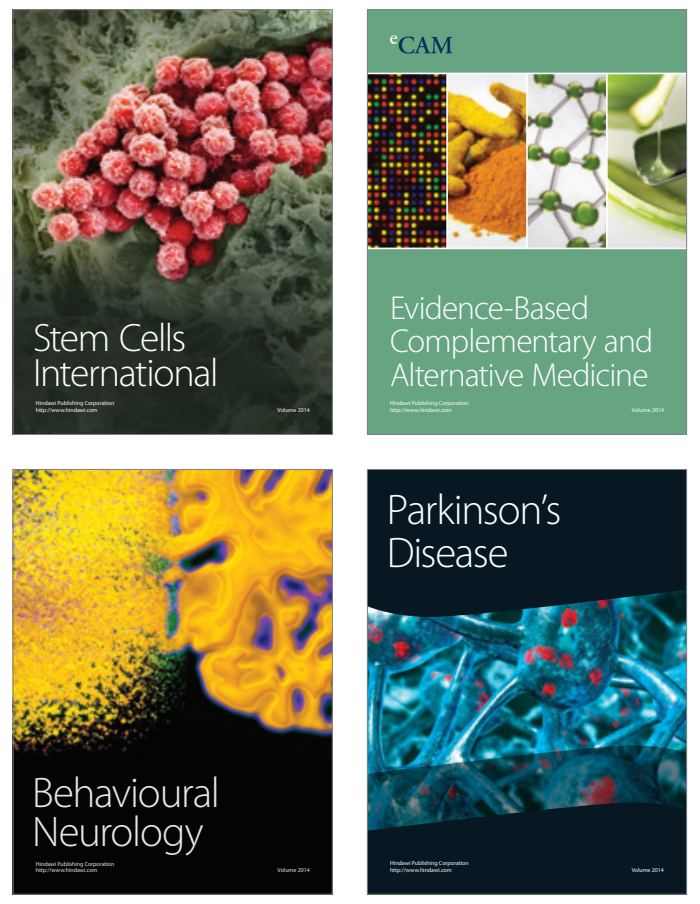
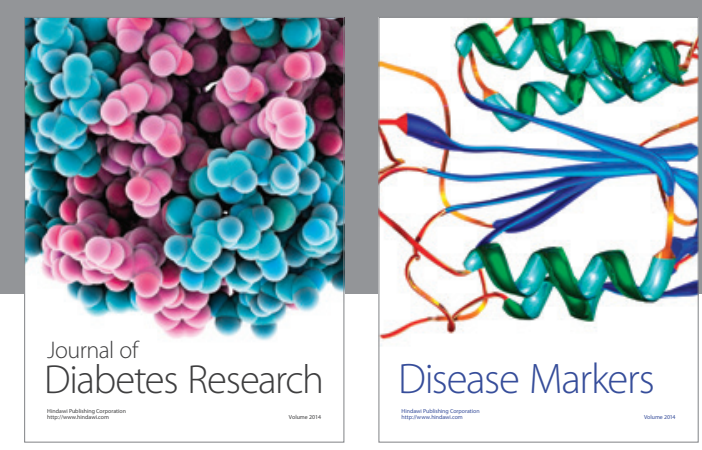

Disease Markers
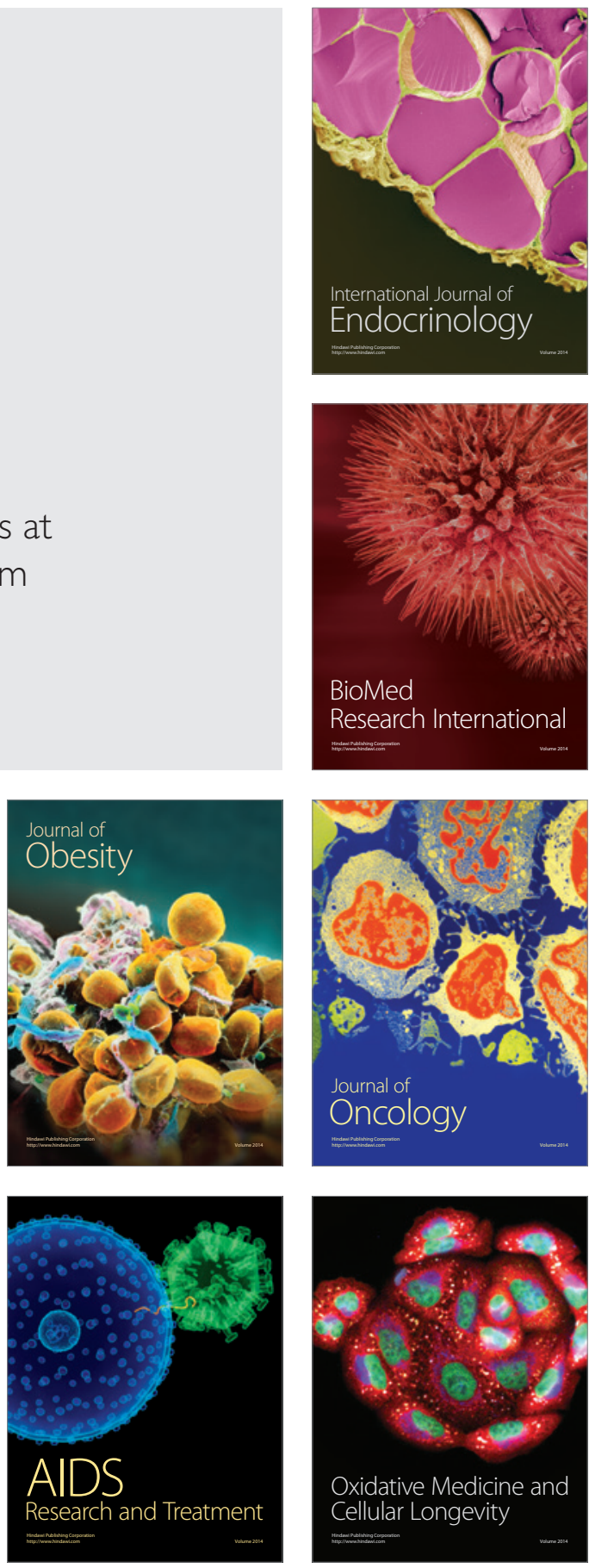